\title{
Las prácticas evaluativas de los docentes de educación física en Villavicencio Evaluation practices of physical education teachers in Villavicencio
}

\author{
Edgar Talero, Nicolás Guarnizo \\ Universidad de los Llanos (Colombia)
}

\begin{abstract}
Resumen. Se hace una descripción de los instrumentos evaluativos que implementan los docentes de Villavicencio a la hora de evaluar aprendizajes. El objetivo de este estudio quiso determinar cuáles son esas prácticas evaluativas que implementan los docentes del área en la ciudad, especialmente el seguimiento a los estudiantes con dificultades de aprendizaje motriz, pues es necesario entender si la evaluación formativa se aleja de la mirada tradicional de clasificar individuos, entre quienes tienen las condiciones y quienes no las tienen, pues la evaluación es un proceso de comprensión y mejora tanto de la enseñanza como del aprendizaje. El tipo de estudio que se implementó fue un enfoque cualitativo y la metodología descriptiva, debido a que se buscaba un conocimiento directo de la realidad, a través de las observaciones que los investigadores realizaron durante las salidas de campo, en las cuáles indagaron el tipo de prácticas evaluativas que los docentes de educación física. Con esta investigación se logró establecer que los docentes no diseñan instrumentos de evaluación que les permitan hacer un seguimiento a los estudiantes que presentan dificultades de aprendizaje.
\end{abstract}

Palabras Claves: aprendizaje motriz, educación física, evaluación tradicional, práctica.

\begin{abstract}
A description is made of the evaluative instruments implemented by Villavicencio teachers when evaluating learning. The objective of this study was to determine what evaluation practices are implemented by teachers in the area in the city, especially the follow-up of students with motor learning difficulties, since it is necessary to understand if the formative evaluation moves away from the traditional view of classifying individuals, between those who have the conditions and those who do not, since evaluation is a process of understanding and improvement of both teaching and learning. The type of study that was implemented was a qualitative approach and descriptive methodology, because a direct knowledge of reality was sought, through the observations that the researchers made during the field trips, in which they investigated the type of evaluative practices than physical education teachers. With this research it was possible to establish that teachers do not design evaluation instruments that allow them to monitor students with learning difficulties.
\end{abstract}

Keywords: motor learning, physical education, traditional assessment, practice.

\section{Introducción}

Es de resaltar la importancia que tiene para los docentes de educación física valorar las conductas motrices de los estudiantes, desde el saber pedagógico del área, un saber que va más allá de la mirada tradicional de la educación física que se ha encontrado, y que está orientada a la medición del cuerpo a partir del rendimiento deportivo de los estudiantes, esa mirada tradicional hace que los docentes se alejen de las tendencias actuales, que anudan esfuerzos desde una evaluación formativa, también conocida como procesual o continua. Por esta razón, se debe lograr una innovación de procesos de evaluación para dar pie a la construcción de aprendizajes significativos en las clases (Jaramillo \& Carballo, 2021, p. 59).

De acuerdo con lo anterior, podemos deducir que la evaluación con carácter formativo ha de orientarse a

Fecha recepción: 05-11-21. Fecha de aceptación: 31-01-22

Edgar Talero

edgar.talero@unillanos.edu.co conocer y comprender como enseñan los docentes y como aprende los estudiantes, Jiménez et al (2021) menciona que:

La didáctica para la evaluación en la Educación Física (EF) es uno de los aspectos más relevantes dentro de la investigación de dicha disciplina pedagógica, incluyendo, por ejemplo, aspectos asociados al tiempo, a la organización del alumnado, al uso del material, a los estilos de aprendizaje y a los estilos de enseñanza (EE) del profesorado. (p.47).

Es por eso que, desde este tipo de estudios, se pretende describir como a nivel didáctico abordan las prácticas evaluativas los docentes de educación física de la ciudad de Villavicencio-Colombia.

Esta investigación se desarrolló teniendo en cuenta los instrumentos bajo los cuales los docentes hacían seguimiento a las dificultades que presentaban los estudiantes que tienen problemas al momento de alcanzar ciertos aprendizajes motrices desde la clase de educación física, el estudio de Serrano y de Luque (2019) se menciona la importancia de realizar una debida evalua- 
ción en los niños frente a la motricidad fina y gruesa, este seguimiento debe ser progresivo para los estudiantes, de este modo, se podrá llevar a cabo un mejoramiento en la acción motriz.

En ese mismo sentido, se comprobó si existían instrumentos de seguimiento, si la evaluación era centrada en procesos educativos o por el contrario enfocada en la valoración de las habilidades físicas o la condición motriz de los estudiantes.

En este orden de ideas, es importante que los docentes de educación física tengan claro como implementar prácticas evaluativas acordes para el área y al nivel de aprendizaje motriz de cada niño, logrando de tal forma, que los procesos evaluativos que implementan, sean acordes con el paradigma cualitativo, pues la evaluación vista como un proceso didáctico a de orientarse a través de una comunicación recíproca entre docente y estudiante. Es importante por lo anterior tener en cuenta que:

Son muchas las formas y los medios de evaluar a los estudiantes en el área de Educación Física pero no hay consenso sobre la forma y sobre que cultura evaluativa debería de imperar en la evaluación. Existe gran disparidad de criterios que se están utilizando para Evaluar la Educación Física en los centros de Educación Infantil y Primaria e Institutos de Secundaria. Por ejemplo, hay profesionales que califican a sus estudiantes en función de unas pruebas físicas que pasan a sus alumnos al inicio y final de curso, otros atienden a criterios meramente motrices, otros a criterios de conocimiento y/o actitudinales, etc. (Calatayud, 2019, p. 259).

Es por ello que en algunas instituciones educativas al evaluar el área de educación física, sigue prevaleciendo la evaluación de corte positivista, que proviene de la parte deportiva y que centra sus esfuerzos sobre todo en: la medición del cuerpo a partir de las condiciones físicas de los estudiantes, todo esto se ve en el uso que hacen los docentes de ciertas baterías de test, con el único objetivo de medir las capacidades condicionales de los estudiantes, desconociendo los distintos niveles de aprendizaje motriz que tiene el estudiante, comparándolo así con un atleta de alto rendimiento. Por otro lado, en el campo de la educación física es necesario contemplar el cuerpo desde lo complejo, lo armonioso y la expresividad (Tabares, 2021).

\section{Una mirada tradicional a la evaluación de la educación física}

Para abordar el presente y ver hacia el futuro, es preciso revisar el pasado para no seguir cometiendo las mismas inconsistencias didácticas del área. Es así como al hablar de evaluación educativa, es imperioso dar una mirada al paradigma evaluativo de carácter tecnológico, positivista y psicotécnico que, bajo el modelo conductista de Ralph Tyler, se afianzó en la pedagogía por objetivos. De igual manera Stufflebeam y Shinkfield (1987), menciona que:

Tyler resumió sus concepciones en cuanto a que el proceso de la evaluación es esencialmente el proceso de determinar hasta qué punto los objetivos educativos han sido actualmente alcanzados mediante los programas de currículo y de enseñanza. De cualquier manera, desde el momento en que los objetivos educativos son esencialmente cambios producidos en los seres humanos, es decir, ya que los objetivos alcanzados producen ciertos cambios deseables en los modelos de comportamiento del estudiante, entonces la evaluación es el proceso que determina el nivel alcanzado realmente por esos cambios de comportamiento (p.92).

Bajo este paradigma educativo, se organizó todo un modelo pedagógico en torno al currículo y la evaluación, Ralph Tyler es considerado el padre de la evaluación educativa, él desarrollo un enfoque netamente conductista sobre cómo habría de concebirse el aprendizaje en la escuela, teniendo en cuenta el paradigma conductista de la psicológica y un esquema de la producción industrial traído a la escuela desde el taylorismo empresarial.

Los test para la evaluación física son fiables y objetivas, pero que de igual manera sirven para la formación deportiva (Vernetta, Montosa \& Gutiérrez, 2019). Resulta claro que, desde una mirada tradicional de la educación física, se tiende a través de pruebas de medida física a clasificar, jerarquizar y seleccionar talentos deportivos.

Es necesario dilucidar que la validez de una prueba indica siempre la manera adecuada como se han de registrar los datos obtenidos al momento de realizarlas, igualmente la fiabilidad se da en la exactitud con el que se miden las capacidades básicas, las habilidades físicas o la condición física de los estudiantes, por último, al momento de aplicar un test; la objetividad de cualquier prueba física se logra al observar el grado de independencia que se da entre el resultado de una prueba y la persona que se está evaluando. Atienza, Valencia y Devís (2018) afirma: «El enfoque tradicional de evaluacióncalificación en EF se corresponde de una forma clara con discursos y enfoques de una EF orientada al rendimiento, así como con un currículum por objetivos (...) 
que se entiende la evaluación como medición, control y/o poder» (p.128).

Para tal efecto, es necesario aclarar que el tipo de pruebas que implementan muchas veces los docentes desde un supuesto saber pedagógico de la educación físi$\mathrm{ca}$, siempre han estado encaminadas a valorar las capacidades básicas, las habilidades físicas o la condición física de los estudiantes; a la hora de medir estas capacidades o habilidades, los docentes recurren a test estandarizados, sin embargo, Sonlleva, Martínez y Monjas (2018) afirman que:

La comunicación en la evaluación entre profesores y estudiantes es uno de los aspectos mejor valorados en las entrevistas realizadas a los docentes de EF, pues la comunicación favorece la progresión del aprendizaje del alumno, motiva el desarrollo de su sentido crítico y reflexivo, así como la mejora de sus habilidades y destrezas en la formación (p. 346).

La afirmación anterior, demuestra que es importante la comunicación durante la evaluación, por esta razón, el docente debe realizar prácticas evaluativas como los test, llevando una comunicación acertada con los estudiantes, esto con el fin de realizar un mejoramiento en las prácticas corporales como la motricidad fina y gruesa, «un test es una prueba determinada que permite la medida en un individuo de una característica precisa comparándola a los resultados obtenidos por otras personas» (Blázquez, 2010, p. 190). En esta perspectiva se trata de una evaluación de tipo normativo que tiende a desconocer las condiciones del contexto en el que está inmerso el estudiante y su nivel motriz, al compararlo con los resultados de una población o un grupo particular.

\section{La evaluación formativa abordada desde las clases de educación física}

La evaluación formativa conocida también como procesual, parte de un proceso sistemático que no solo pretende aprobar o reprobar estudiantes clasificándolos, sino por el contrario busca la apropiación y dominio de conocimientos por parte de los mismos, logrando así, que ellos se sientan motivados a participar dentro de su proceso de aprendizaje. Huamaní (2016) señala que: «la evaluación procesal no solo es un instrumento para el docente, lo es también para el alumno, porque le permite alcanzar habilidades que exige la sociedad del conocimiento como aprender a prender y generar conocimiento.» (p. 35).

Resulta pertinente aclarar, que para que este pro- ceso sea exitoso, los instrumentos le deben dar viabilidad y fiabilidad a las decisiones que se tomen a partir de los datos recogidos y analizados desde una evaluación formativa, pues esta permite que el docente ajuste sus objetivos de enseñanza, de acuerdo a los ritmos de aprendizaje de sus estudiantes. Según Hoffman (2011) afirma que:

É importante a mediação e abordagem, o diálogo, o acompanhamento no modo de ser e no aprendizado de cada aluno, apertando as mãos com rigor e carinho, para ajudá-lo a continuar sempre, tendo a opção de escolher as direções em seu processo de aprendizagem. (p. 50) (Es importante mediar y abordar, dialogar, acompañar en la forma de ser y en el aprendizaje de cada alumno, estrechándole la mano con rigor y afecto, para ayudarlo a continuar siempre, teniendo la opción de elegir las direcciones en su proceso de aprendiendo).

Cabe agregar, que para las tendencias actuales que orientan la evaluación en educación física al servicio de los aprendizajes motrices de los estudiantes, la observación es un instrumento muy valioso para determinar lo que sucede en la clase de educación física; no obstante, el uso de estas herramientas o instrumentos permiten el contraste del nivel de aprendizaje motriz, observando el logro o el éxito del estudiante (Pueyo \& Alcalá, 2020).

En efecto los docentes no pueden seguir confundiendo pruebas de carácter físico, con procesos de evaluación formativo, siendo importante aclarar que el problema no está en la utilización de pruebas de medida física a la hora de evaluar en la clase de educación física, sino en las decisiones que se toman a partir de los resultados arrojados por estas pruebas, estos resultados no pueden estar orientadas a la selección por vía del etiquetado, «en la última escuela, donde no hay «yo» sin los otros, las etiquetas no existen.» (Bona, 2017, p. 168).

Las pruebas de medida física «test» no pueden ser implementadas para etiquetar a quienes no logran alcanzar el baremo propuesto en la prueba, o para que desde las clases de educación física se seleccionen a los más aptos motrizmente y se excluya a aquellos que tienen dificultad en el proceso de aprendizaje. Autores como Álvarez, (2011) menciona que: «Una educación entendida como proceso de selección y de exclusión restringe las posibilidades de acceder al conocimiento, y acarrea consecuencias directas sobre el currículo y su implementación» (p. 58).

Se observa claramente que la principal característica de la evaluación formativa en educación física, es contar con instrumentos que permitan observar las con- 
ductas motrices de los estudiantes y también otros contenidos en la formación del discente, estos instrumentos les permiten a los docentes hacer un seguimiento de los estudiantes que presentan dificultades en el aprendizaje, es preciso aclarar que es un instrumento de evaluación en educación física:

El instrumento de evaluación es la herramienta que permite recoger información del logro de aprendizaje (o no). Durante mucho tiempo, los test estandarizados y las pruebas objetivas han constituido los instrumentos más usados para la evaluación de la educación física, en un enfoque por competencias, los procedimientos de apreciación directa toman protagonismo a la hora de juzgar los aprendizajes logrados por el alumnado (González \& Lleixà, 2010, p. 130).

Del anterior planteamiento se deduce que los docentes al momento de implementar una evaluación formativa o procesual, tienen que partir de unos interrogantes pedagógicos que le den un norte a su quehacer, estos interrogantes son: ¿Qué conocimientos quiero que los estudiantes reciban? ¿Qué estrategias se deben utilizar para que los estudiantes se apropien de los aprendizajes motrices de la clase? Es decir que el docente debe de ser minucioso en la elaboración de instrumentos evaluativos que le ayuden a tomar decisiones didácticas, para que durante un periodo o semestre, los estudiantes alcancen las competencias y mejoren sus capacidades físicas y coordinativas. Cabe mencionar que al utilizar técnicas como la observación posibilita el proceso de una evaluación formativa, esta técnica tiene siempre presente el entorno socio-cultural de los estudiantes, es eso lo que dice el racionalismo sobre ser consecuente con el otro, partiendo de las dificultades que tiene el estudiante al momento de aprender, así, Pérez et al (2017) afirma:

hace referencia a la que se lleva a cabo en el aula de forma diaria y cotidiana, normalmente con una finalidad formativa, recopilando sistemáticamente información del proceso de aprendizaje de cada alumno. En consideración de los autores, este tipo de evaluación, también se considera como sistemática o frecuente, aunque no es necesario que se le otorgue una calificación cuantitativa (p. 270).

Cabe decir, que al momento de establecer los objetivos y competencias para la clase, el docente desde procesos formativos toma decisiones, que le llevan a establecer los contenidos que le permitirán al estudiante alcanzar las competencias planteados para el curso, pues el docente tiene claro cuáles son las habilidades, destrezas o capacidades físicas que tiene que alcanzar el estudiante la evaluación «habría de estar referida por una parte al desarrollo de la competencia motriz y por otro al desarrollo de la condición física con especial referencia a la adaptación del esfuerzo» (Onofre, 2006, p. 305). Así la información suministrada por las pruebas no puede referirse únicamente a cuánto aprende el alumno por ejemplo en cuanto a velocidad, lo que ocurriría si sólo se utiliza test o pruebas, sino que debe estar referido a cómo los alumnos aprenden los conocimientos y los dotan de significado, para lo que se precisa una vigilancia sistemática del proceso de enseñanza - aprendizaje.

Ahora bien, la evaluación formativa se enfoca en los procesos de enseñanza-aprendizaje, estos procesos constituyen el foco de toda evaluación formativa, ya que este momento de la evaluación, lleva a que los docentes tomen decisiones encaminadas a comprender y mejorar la comunicación didáctica entre docente- discente.

Para finalizar, es importante definir que la evaluación no es un proceso didáctico que se limita a controlar, clasificar, seleccionar, sancionar, reprobar o etiquetar a los estudiantes, todo lo contrario, es un proceso que suministra información oportuna a los docentes, para que logren tomar decisiones de tipo didáctico que le permitan diagnosticar a tiempo dificultades que se presentan dentro de los procesos de enseñanza - aprendizaje y así lograr el mayor objetivo de cualquier docente, que es, que sus estudiantes logren alcanzar las competencias del curso y aprobarlo. Por este motivo el propósito del artículo se esboza en la búsqueda de los resultados tangibles en el campo de la evaluación en el área de la educación física.

Por esta razón el objetivo principal del estudio quiso determinar cuáles son esas prácticas evaluativas que implementan los docentes del área en la ciudad, especialmente el seguimiento a los estudiantes con dificultades de aprendizaje motriz.

\section{Metodología}

Esta investigación propuso un enfoque metodológico de tipo cualitativo, «la investigación es un proceso sistemático a través del cual se trata de alcanzar, por medio de información y datos, la respuesta a una pregunta, la solución a un problema; o bien, un mayor entendimiento de un fenómeno» (Daza, 2018, p. 94).

La investigación se implementó desde el método descriptivo, en razón a que se muestra de manera sistemática cuáles son las prácticas evaluativas que implementan los docentes de educación física de la ciu- 
dad de Villavicencio, para orientar los procesos de enseñanza-aprendizaje. El estudio se orientó desde el diseño fenomenológico, por lo que se necesita estudiar, observar y describir sus propiedades, implicando el colectivo de personas, en el caso del ámbito educativo, se estudia las áreas relacionadas, como la evaluación, la pedagogía, la didáctica, currículo, etc. (Daza, 2018).

\section{Participantes}

La población objeto de estudio fue de 100 docentes del área de educación física en nivel de primaria, pertenecientes a la secretaría de educación del municipio de Villavicencio, la obtención de la muestra, para el desarrollo de la presente investigación, se dio a partir de una muestra por conveniencia de 32 docentes. Es necesario aclarar que los individuos del muestreo por conveniencia se seleccionaron porque eran quienes estaban disponibles a participar durante el desarrollo del trabajo.

\section{Instrumentos}

En la medida en que se fueron obteniendo y construyendo los datos a partir de técnicas de recolección como la observación no participante, puesto que «Al analizar y procesar, se hace una selección particular de la información y se apoya no solo en la experiencia de vida, nuestro mundo sociocultural, sino también en la intuición y, fundamentalmente, en los propósitos de premisas del estudio» (Bracho et al, 2021, p. 125).

Se aplicó una encuesta guiada, por lo que Katz, Seid y Abiuso (2019) expresan que la encuesta «permite indagar sobre múltiples temas de los individuos o grupos estudiados: hechos, actitudes, creencias, opiniones, pautas de consumo, hábitos, prejuicios predominantes e intenciones de voto» (p. 2). Por lo que se vuelve pertinente para los estudios de índole educativo, en este caso a profundiza el tema de la evaluación.

Por otro lado se hizo hincapié a la revisión documental de los archivos de los docentes: mallas curriculares, sistemas integrados de evaluación de las instituciones educativas donde laboran los docentes y los planes o rutas de aula, «su objetivo principal es dirigir la investigación desde dos aspectos, primeramente, relacionando datos ya existentes que proceden de distintas fuentes y posteriormente proporcionando una visión panorámica y sistemática de una determinada cuestión elaborada en múltiples fuentes dispersas a su vez, se utilizó la técnica de la observación» (Reyes y Carmona, 2020 (p.1).

Para la confiablidad de los instrumentos se tuvo en cuenta la revisión de expertos en el área, que hicieron los debidos ajustes para luego lograr la práctica de campo. Los revisores dieron su viabilidad para generar la recolección de información, contribuyendo en el logro de los hallazgos.

En este orden de ideas, durante un periodo de tres meses se realizaron observaciones con diario de campo a las prácticas evaluativas que realizaban los 32 docentes y se les aplicó una encuesta. Cabe aclarar que las observaciones se hicieron tres veces por semana a los 32 docentes y la encuesta tuvo su objetivo profundizar sobre las prácticas evaluativas que se realizaban en el aula de clase. Posteriormente, para su análisis se triangularon los datos recolectados: el análisis de diarios de campo, la revisión documental y la encuesta, a partir de esto, se logró establecer las relaciones mutuas entre los instrumentos aplicados, al comparar y contrastar la información dentro del proceso investigativo.

El procedimiento para el análisis de la información estuvo enmarcado en el software Atlas Ti, siendo un programa de carácter cualitativo, y con los parámetros de las categorías de análisis, se puedo evidenciar los resultados de este estudio.

\section{Resultados}

Para la presentación de los resultados se procedió a un análisis de tipo cualitativo, a partir de la triangulación de la información recogida a través de la revisión documental, un diario de campo y un cuestionario. La información recogida a partir de estos instrumentos, permitió comparar y contrastar los datos, para de esta forma, determinar cuáles eran las prácticas evaluativas que realizaban los docentes de educación física de la ciudad de Villavicencio desde su racionalidad pedagógica.

- Se logró determinar al contrastar la información que los docentes no hicieron entrega de los planes de aula, por lo cual es difícil creer que estos diseñen instrumentos de evaluación motriz. Cabe mencionar que en el análisis de la información recogida a partir de la revisión documental se confirmó que ninguno de los docentes pertenecientes a la muestra tenían dentro de sus archivos instrumentos de observación motriz.

Además, a partir de las observaciones y la encuesta realizadas a la población docente, ellos aseguraron implementar instrumentos de medida física (test motores) estandarizados, pero aseguraron que dan prioridad al uso de la planilla de notas y la lista de asistencia como instrumentos de evaluación, siendo estas planillas documentos institucionales y no instrumentos de eva- 
luación que permitan hacer un seguimiento cualitativo o cuantitativo a las conductas motrices y a las capacidades físicas de los estudiantes de las instituciones públicas de la ciudad de Villavicencio.

- Se puede señalar que al tratar de identificar la diferencia entre una evaluación educativa o deportiva centrada en la medición del cuerpo a partir de la condición física de los estudiantes, en el municipio deVillavicencio, los docentes no tienen claridad entre la diferencia de una y otra, en razón a que creen utilizar instrumentos estandarizados o pruebas objetivas a los que les modifican los baremos y lo cual fue comprobado mediante la revisión documental, tampoco se encontraron instrumentos de observación motriz o de verificación.

- Se evidenció en el análisis de cada uno de los instrumentos que los docentes que pertenecían a la muestra del estudio aplican instrumentos de medida física, por lo cual logro definirse, que el trabajo evaluativo, desde el saber pedagógico de los docentes, no se está llevando desde una mirada educativa, tampoco desde un enfoque deportivizado que se oriente a la medición del cuerpo a partir de las condiciones físicas de los estudiantes; esto se concluyó porque los docentes no diseñan instrumentos de evaluación que permitan hacerle seguimiento a las capacidades físicas o a las conductas motrices de los estudiantes

Es así como los docentes pertenecientes al estudio evalúan bajo una técnica de observación completamente subjetiva carente de rigurosidad, se encuentran alejados de un paradigma educativo que permita llevar un proceso sistemático en el que la recolección de datos permita a los docentes tomar decisiones sobre los ritmos de enseñanza - aprendizaje de sus estudiantes.

Se estableció cuáles eran los tipos de capacidades y habilidades motrices que valoran los docentes de educación física de la ciudad de Villavicencio, fue así como se determinó que los docentes privilegian la aplicación de test para valorar capacidades condicionales como la fuerza, la resistencia y la velocidad, pero no son test estandarizados, debido a que han modificado los baremos, no aplica ningún instrumento de observación para valorar las conductas motrices de sus estudiantes, y le dan más valor a la planilla de notas que a un instrumento de observación motriz; los docentes a la hora de valorar las capacidades físicas de los estudiantes, dan prevalencia a las capacidades físicas condicionales por encima de las coordinativas.

- Inicialmente, al comparar y contrastar los datos, a partir de las observaciones con diario de campo. Se observó que los docentes por la falta de sistematicidad del proceso, mediante la construcción e implementación de instrumentos evaluativos, no permite individualizar los procesos de aprendizaje motriz y así lograr adaptarlos a las necesidades de los estudiantes, es claro que la sistematización de los procesos de enseñanza- aprendizaje se dan desde los datos obtenidos en el proceso evaluativo, estas decisiones deben ser de tipo pre-activo, interactivo y post-activo a partir de los análisis del proceso evaluativo, los cuales han de ser orientados de acuerdo con los ritmos de aprendizaje de los estudiantes.

\section{Discusión y conclusiones}

Los resultados de este estudio han mostrado, concretamente que los datos obtenidos en proceso evaluativos realizados por los docentes de la ciudad de Villavicencio carecen de instrumentos evaluativos que les permitan hacer seguimiento al proceso de aprendizaje de los estudiantes en el nivel secundario, pues los docentes del área de Educación Física carecen de instrumentos de medida física o de observación, lo que muestra que han limitado la evaluación a un proceso subjetivo que se limita a la planilla de notas y a la de asistencia; difícilmente se puede determinar cómo realizan una evaluación para mejorar el aprendizaje motor de los estudiantes.

Con base en lo anterior, es difícil identificar cómo los docentes logran evaluar la condición física de sus estudiantes al no contar con pruebas estandarizadas, Esto quiere decir que no valoran de manera objetiva las cualidades motrices de los estudiantes, para el análisis de la condición física se deben utilizar instrumentos que evalúan la aptitud en todas sus dimensiones. Un ejemplo en Europa es la batería EUROFIT. Esta batería tiene como objetivo valorar de manera sencilla, valida, rápida, segura y económica los principales factores de la condición física relacionados con la salud (Cruz, et al., 2014).

Otro de los hallazgos encontrados a partir de la triangulación de los datos obtenidos en los instrumentos, es que los docentes no están construyendo instrumentos de observación para hacer seguimiento a los ritmos de aprendizaje que presentan los estudiantes en las clases, pues los resultados obtenidos en la encuesta guiada muestran que la mitad de los docentes no usan ningún tipo de prueba o instrumento que de muestra de una planificación o sistematicidad del proceso evaluativo, es claro que el docente debe centrar sus esfuerzos en la valoración de aprendizajes procedimentales sin dejar de lado lo conceptual y actitudinal, pues la evaluación 
debe ser un proceso integral, centrando claro está los análisis a partir del desarrollo de la motricidad de los estudiantes. En el estudio de Peña et al, (2019) establecieron que se busca reemplazar la visión reduccionista de la evaluación del rendimiento, por una evaluación que abarque el conjunto de aspectos, procesos y variables involucradas en las actividades escolares, en definitiva, se intenta llegar a una mirada integral de la evaluación en EF.

Es importante añadir que la evaluación está ligada a las decisiones de tipo formativo que tome el docente a partir de los resultados que arroje el proceso de valoración, pues estas decisiones se tienen que encaminar a la mejora de los procesos de enseñanza -aprendizaje. Las prácticas evaluativas al interior de las clases de educación física no pueden limitarse a la implementación exclusiva de instrumentos de medida física, es necesario recurrir a instrumentos de verificación y observación según lo percibido en las instituciones educativas de la ciudad de Villavicencio. Autores como Rodríguez, Curilem, Escobar y Valenzuela (2016) señalan que «debería existir menor atención al rendimiento físico de los estudiantes y valorar de forma integral sin fragmentar los conocimientos y las actitudes de los estudiantes» (p. 31).

Con el trabajo de investigación se estableció que los docentes no diseñan instrumentos de evaluación que les permita hacer un seguimiento procesual a los estudiantes que presentan dificultades a la hora de realizar tareas motrices en clase, pues el proceso evaluativo se encuentra orientado desde los contenidos de la clase a valorar simple y exclusivamente las capacidades físicas de los estudiantes. para Blázquez (2017):

los instrumentos son recursos que se emplean para recolectar la información y deben poseer ciertas condiciones para que se garantice una evaluación acorde con las intenciones. constituyendo un medio para obtener información y por tanto influye en la construcción de la realidad a evaluar (p.197).

Al contrastar los datos recogidos sobre el desarrollo de las habilidades o capacidades físicas, se determinó que los docentes siguen bajo un paradigma en el que prima la medición del cuerpo a partir del rendimiento deportivo, los docentes usan el deporte como un fin y no como un medio para el desarrollo de las capacidades físicas, no podemos olvidar, que la mejora de la calidad de las clases de E.F. y no puede perderse el objetivo de la disciplina no debe centrarse en el rendimiento deportivo del estudiante (Flintoff, Foster \& Wystawnoha, 2011), sino que, debe existir un impacto desde lo cuali- tativo en la formación de los estudiantes y profesores. Una muestra de esto es que prima por parte de los docentes de la ciudad de Villavicencio el desarrollo de las capacidades condicionales como: la fuerza, la velocidad y la resistencia, pues es fácil de constatar a partir de las pruebas de medida física que implementan para valorar las capacidades condicionales, contrario a lo anterior, no hay evidencia de instrumento alguno que permita valorar las capacidades coordinativas o las conductas motrices de los estudiantes.

\section{Referencias}

Álvarez, J. (2011). Evaluar para conocer, examinar para excluir. Madrid: Morata.

Atienza, R., Valencia, A., \& Devís, J. (2018). Experiencias de Evaluación en Educación Física. Una Aproximación desde la Formación Inicial del Profesorado. Estudios pedagógicos (Valdivia), 44(2), 127-147. DOI doi.org/10.4067/s0718-07052018000200127

Blázquez, D. (2010). Evaluar en educación física. Barcelona: Inde.

Blázquez, D. (2017). Cómo evaluar bien educación física, el enfoque de la evaluación formativa. Barcelona: Inde.

Bona, C. (2017). Las escuelas que cambian el mundo. Educatio Siglo XXI, 35(1), 165-172.

Bracho, M. S., Fernández, M., \& Díaz, J. (2021).Técnicas e instrumentos de recolección de información: análisis y procesamiento realizado por el investigador cualitativo. Revista Científica UISRAEL, 8(1), 107121.

Calatayud Salom, M. A. (2019). Una oportunidad para avanzar hacia la evaluación auténtica en Educación Física. Retos: Nuevas Perspectivas de Educación Física, Deporte y Recreación, (36), 259-265. DOI doi.org/ 10.47197/retos.v36i36.67540

Cassanova, M. (1997). Manual de evaluación educativa. Madrid: La Muralla.

Chaverra, B.E., \& Hernández, J.L. (2018). La planificación de la evaluación en educación física: Estudio de casos sobre un proceso desatendido en la enseñanza. Revista Electronica Educare. 23, (1). DOI 10.15359/ ree.23-1.12

Cruz, A., Lara, A. J., Zagalaz, M. L., \& Torres, G. (2014). Análisis y evaluación de la condición física en estudiantes de educación primaria de un medio rural y urbano. Apunts: Educació Física I Esports, 116, 44-51. DOI 10.5672/apunts.2014-0983.es.(2014/2).116.04

Daza, W. G. I. (2018). Investigación educativa desde un enfoque cualitativo: la historia oral como método. 
Voces de la Educación, 3(6), 93-110.

Flintoff, A., Foster, R., \& Wystawnoha, S. (2011). Promover y mantener la educación física y el deporte escolar de alta calidad a través de asociaciones deportivas escolares. Revista europea de educación física, 17 (3), 341-351. DOI doi.org/10.1177/ $1356336 \mathrm{X} 11416731$

Gonzales, C., \& Lleixà. T. (2010). Didáctica de la educación física. Barcelona: GRAÓ

Hoffman, J. (2011). Avaliar para promover as setas do caminho. Porto Alegre: Mediação.

Huamaní, L. (2016). Efectos de la evaluación procesal en la calidad de la gestión del conocimiento de los estudiantes del tercer año del curso de geografía del Colegio de Aplicación San Marcos. (Proyecto de grado). Universidad Nacional Mayor de San Marcos.

Jaramillo, E. T., \& Carballo, N. G. (2021). El currículo de la educación física en modalidad e-learnig y blearning durante el confinamiento. Acciónmotriz, (27), 57-65.

Jiménez, E., Ramírez, D., Torres, B., Santander, I., Uribe, N., \& Mujica, F. (2021). Estilos de enseñanza en Educación Física escolar en el contexto de pandemia:Teaching styles in school physical education in the context of pandemics. Revista Académica Internacional de Educación Física, 1(4), 46-57.

Katz, M., Seid, G., \& Abiuso, F. L. (2019). La técnica de encuesta: Características y aplicaciones. Universidad de Buenos Aires. Argentina.

Onofre, R., y Contreras, J. (1998). Didáctica de la educación física un enfoque constructivista. Barcelona: Inde.

Peña, S., Toro, S., Osses, S., Pachón, J., \& Hernández, C. (2019). La Dimensión Conceptual del Conocimiento en Educación Física: Un estudio Binacional. Retos: nuevas tendencias en educación física, deporte y recreación, (35), 170-175. DOI doi.org/10.47197/ retos.v0i35.63164

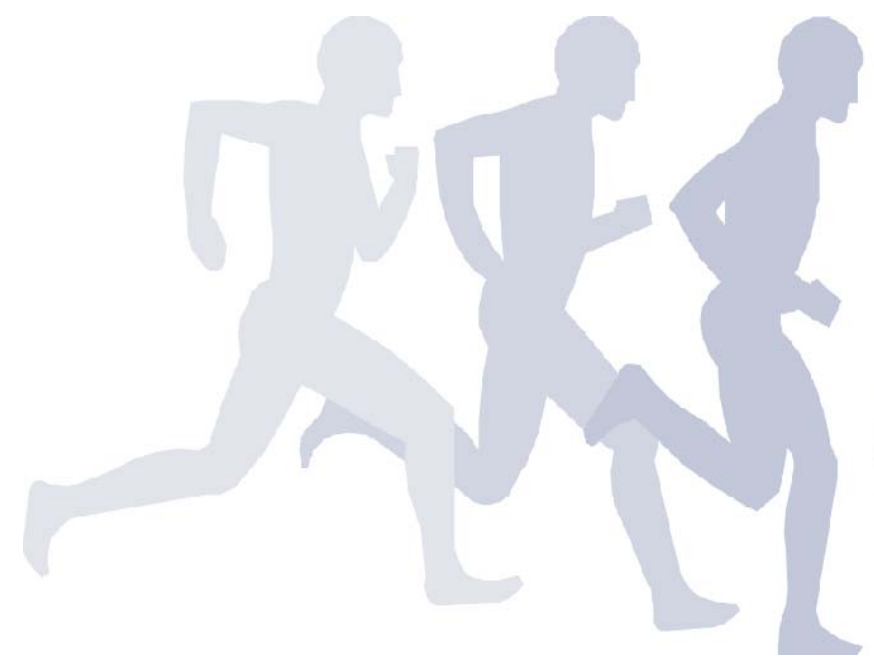

Pérez, M., Enrique, J. O., Carbó, J. E., \& González, M. (2017). La evaluación formativa en el proceso enseñanza aprendizaje. Edumecentro, 9(3), 263-283.

Pueyo, Á. P., \& Alcalá, D. H. (2020). ¿Y si toda la innovación no es positiva en Educación Física?: Reflexiones y consideraciones prácticas. Retos: nuevas tendencias en educación física, deporte y recreación, (37), 579587. DOI doi.org/10.47197/retos.v37i37.74176

Reyes-Ruiz, L. \& Carmona Alvarado, F. A. (2020). La investigación documental para la comprensión ontológica del objeto de estudio.

Rodríguez, F., Curilem, C., Escobar, D., \& Valenzuela, L. (2016). Propuesta de evaluación de la educación física escolar en Chile. Educación Física y Ciencia, 18(1), 00-00. DOI doi.org/10.35376/10324/33110

Sales, J. (2001). La evaluación de la educación física en primaria una propuesta práctica. Barcelona: Inde

Serrano, P., \& de Luque, C. (2019). Motricidad fina en niños y niñas: desarrollo, problemas, estrategias de mejora y evaluación (Vol. 84). Narcea Ediciones.

Sonlleva, M., Martínez, S., \& Monjas, R. (2018). Los procesos de evaluación y sus consecuencias. Análisis de la experiencia del profesorado de Educación Física. Estudios pedagógicos (Valdivia), 44(2), 329-351. DOI doi.org/10.4067/S0718-07052018000200329

Stufflebeam, D. L., \& Shinkfield, A. J. (1987). Evaluación sistemática: guía teórica y práctica (No. 371.26 S933e). Paidós.

Tabares, G. (2021). Didáctica de la educación corporal. Revista Académica Internacional de Educación Física, 1(1), 21-30.

Vernetta Santana, M., Montosa, I., \& Gutiérrez-Sánchez, Á. (2019). Validación y fiabilidad de un test para evaluar la coordinación óculo manual y agilidad en gimnasia rítmica. Sportis, 5(2), 174-189. DOI doi.org/ 10.17979/sportis.2019.5.2.3488

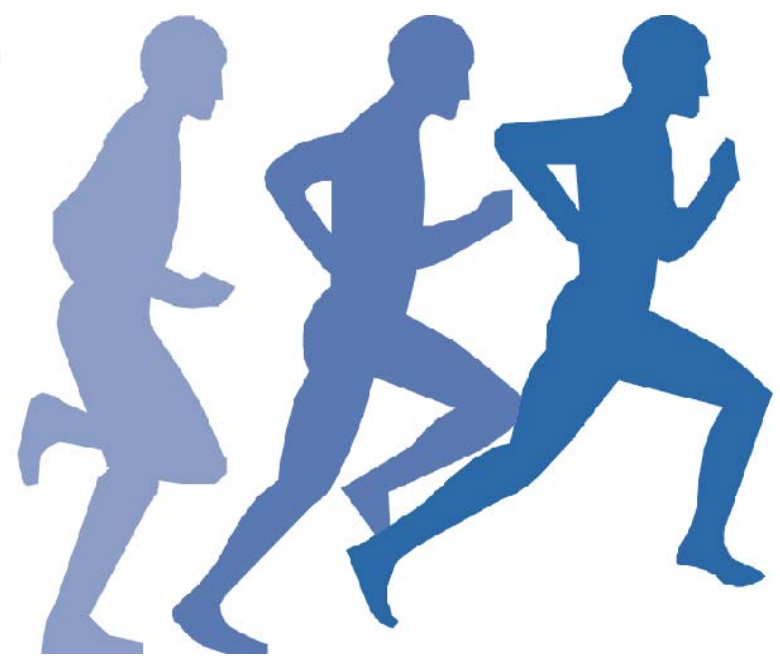

